\title{
Advantage of beam angle optimization in head-and-neck IMRT: patient specific analysis
}

\author{
Tiago Ventura ${ }^{1,2,3[0000-0003-1562-0387]}$, Maria do Carmo Lopes ${ }^{1,2,3[0000-0001-7339-7342]}$, \\ Humberto Rocha ${ }^{3,4[0000-0002-5981-4469]}$, Brigida da Costa Ferreira ${ }^{3,5[0000-0001-7988-7545]}$ and \\ Joana Dias ${ }^{3,4[0000-0003-2517-7905]}$ \\ ${ }^{1}$ Physics Department of University of Aveiro, Campus Universitário de Santiago, 3810-193 \\ Aveiro, Portugal \\ ${ }^{2}$ Medical Physics Department of Instituto Português de Oncologia de Coimbra Francisco \\ Gentil, EPE, Avenida Bissaya Barreto, no 98, 3000-075 Coimbra, Portugal \\ ${ }^{3}$ Institute for Systems Engineering and Computers at Coimbra, Rua Sílvio Lima, 3030-290 \\ Coimbra, Portugal \\ ${ }^{4}$ Economy Faculty of University of Coimbra and Centre for Business and Economics \\ Research, Avenida Dias da Silva 165, 3004-512 Coimbra, Portugal \\ ${ }^{5}$ School Health Polytechnic of Porto, Rua Dr. António Bernardino de Almeida, 400, 4200-072 \\ Porto, Portugal \\ tiagoventuradipocoimbra.min-saude.pt
}

\begin{abstract}
Radiation therapy (RT) main purpose is to eliminate, in a controlled way, all tumor cells sparing as much as possible the normal tissues. IntensityModulated Radiation Therapy (IMRT) is becoming the standard treatment technique in RT. Beam angle optimization (BAO) has potential to confer more quality to IMRT inverse planning process compared to manual trial and error approaches. In this study, the BAO advantages in head-and-neck patients are highlighted, using a patient specific analysis. Fluence optimization was done with Erasmus-iCycle multicriterial engine and $\mathrm{BAO}$ optimization was performed using two different algorithms: a combinatorial iterative algorithm and an algorithm based on a pattern search method. Plan assessment and comparison was performed with the graphical tool SPIDERplan. Among a set of forty studied nasopharynx cancer cases, three patients have been select for the specific analysis presented in this work. BAO presented plan quality improvements when beam angular optimized plans were compared with the equidistant beam angle solution and when plans based on non-coplanar beams geometries were compared with coplanar arrangements. Improvement in plan quality with a reduced number of beams was also achieved, in one case. For all cases, BAO generated plans with higher target coverage and better sparing of the normal tissues.
\end{abstract}

Keywords: Radiation therapy, beam angle optimization, plan assessment, headand-neck cancer. 


\section{Introduction}

Radiation therapy (RT) is one of the most important therapeutic options used in the battle against cancer. It makes use of ionizing radiation to eliminate in a controlled way the tumour cells, sparing as much as possible the adjacent normal tissues. IntensityModulated Radiation Therapy (IMRT) is a radiation therapy technique that enables the generation of conformal dose distributions to the target volume by delivering nonuniform intensity fields from multiple directions. IMRT treatment planning uses inverse planning techniques, wherein an objective function containing the desired plan objectives guides the fluence map optimization (FMO) by scoring the goodness of the plan [1]. The FMO will determine the beam intensities for each of the selected angles. Most of the times, an empirical trial-and-error manual tuning of plan parameters (like weights, objectives or beam angles) is done until an acceptable plan is achieved. This planning framework is extremely dependent on the planner skills and experience and on the case complexity [2]. Moreover, it is not possible to guarantee that an optimal plan is found. The IMRT optimization process should be tackled by more reliable methods and algorithms such as multi-criteria optimization, beam angle optimization (BAO) and eventually by machine learning automated techniques.

BAO methods have contributed to the enhancement of the IMRT optimization process. A beam angle selection based on mathematical criteria may lead to important improvements in the quality of the plan dose distribution [3] that can be even more expressive if non-coplanar beam geometries are available in the optimization process [4]. The BAO problem mathematically described as a highly non-convex multi-modal optimization problem with many local minima [5] can be addressed separately or jointly with the FMO problem. For the coupled modality, the FMO solution guides the BAO problem along the optimization [6], where the best beam ensemble can be achieved using heuristic methods in exhaustive combinatorial searches over a discretized space search $[6,7,8]$ or alternatively using pattern search methods [9] or multistart derivativefree optimization frameworks [4] that continuously explore the search space.

The purpose of this work is to highlight the advantages of BAO for the head-andneck pathology following a patient specific analysis approach. From a set of forty nasopharyngeal (NPC) studied cases, particular patient cases were selected to show the improvements that can be obtained in plan quality when BAO plans are compared with the equidistant beam angle solution and when coplanar and non-coplanar BAO sets are confronted. To complete this patient specific analysis, a third case where it is possible to get improved plans diminishing the number of beams is also evaluated, to emphasize how the individual patient anatomy may influence the results.

\section{Materials and methods}

For this study, three cases were selected among a set of forty NPC clinical cases, previously studied. All cases had a simultaneous integrated boost prescription composed of two dose levels, where the tumour planning target volume (PTV) was prescribed with a dose of $70 \mathrm{~Gy}$ and the lymph nodes PTVs with a dose of 59.4 Gy. 
Spinal cord, brainstem, chiasm, optical nerves, retinas, lens, parotids, oral cavity, larynx, oesophagus, ear canals, temporal mandibular joints, mandible, brain, pituitary gland, thyroid and lungs were also contoured and defined as organs-at-risk (OAR).

IMRT optimization was performed by Erasmus-iCycle, a multicriterial dose calculation engine guided by a wish-list defined a priori, that automatically generates a single Pareto solution for a given set of beams [10]. All plans were initially optimized using the most clinically used beam angle configuration: 7 coplanar equidistant beams $(d 7)$. BAO was performed for coplanar and non-coplanar beam geometries of 5, 7 and 9 beams with two algorithms. The first (algorithm $i$ ) is included in Erasmus-iCycle and it is based in a discrete iterative combinatorial approach [10]. The second algorithm, named as algorithm $B$, belongs to the continuous search space class and uses a pattern search method to find the best possible beam angle ensemble [9].

For each selected NPC case, three plans were considered that highlight the BAO advantages. For the first case (patient \#1), the $d 7$ plan was compared with the 9 noncoplanar beams plan of algorithm $B(B 9 n c)$. For the second case (patient \#2), the 9 coplanar beams plan and the 9 beams non-coplanar plan of algorithm $i$ (i9c and $i 9 n c$, respectively) were compared side-by-side. For the third case (patient \#3), the 5 noncoplanar beams plan of algorithm $B(B 5 n c)$ and the 9 coplanar beams plan of algorithm $i$ (i9c) were compared.

SPIDERplan, a graphical method based on a scoring approach [11], was used for plan assessment and comparison. Targets and OARs are divided into groups. A score based on pre-defined planning objectives and relative weights defined according to the radiation oncologist clinical preferences is assigned to each structure. Global plan quality is computed as a weighted sum of all structures' scores. Graphically, a customised radar plot is used to represent all dosimetric information, where plan evaluation can be done visualizing all structures, in a Structures Plan Diagram (SPD), or just considering the defined groups, in a Group Plan Diagram (GPD). Each group can also be assessed with more detail in partial group radar plots named Structures Group Diagrams (SGD), where only the structures belonging to the group and a partial group score are displayed.

For this study, SPIDERplan configuration included six groups of structures: the PTV group composed by the PTVs, the Critical group constituted by the spinal cord and the brainstem, the Optics group including the chiasm, the optical nerves, the retinas and the lens, the DigestOral group composed by the parotids, the oral cavity, the oesophagus and the larynx, the Bone group composed of the temporal mandibular joint, the mandible and the ear canals and Other group considering the brain, the pituitary gland, the thyroid and the lungs. The groups were assigned with relative weights of $50 \%, 30 \%$, $10 \%, 5 \%, 3.5 \%$ and $1.5 \%$, respectively. The score of each structure is given by the ratio between the clinical tolerance criteria and the planned dose. A score value of one is achieved when the planned dose is equal to the structure tolerance criteria. Higher score values mean that these tolerances were surpassed and the best plans will have scores less than one, tending towards the centre of the radar plot [11]. 


\section{$3 \quad$ Results}

Global plan comparison was done with SPIDERplan GPDs (left diagrams in Fig. 1, Fig. 2 and Fig. 3), while individual group evaluation was performed with SPIDERplan SGDs (right diagrams in Fig. 1, Fig. 2 and Fig. 3). The selection of the groups to be depicted in each figure was based on the visual analysis of the GPD and on the corresponding relative weight importance value.

For patient \#1, $d 7$ and $B 9 n c$ plans were compared, Fig. 1. B9nc plan achieved the best global plan score with an expressive percent difference of $-7 \%$ from $d 7$ score. The largest difference between the tolerance and the planned dose of these plans was obtained for the Optics group. For $d 7$ plan the score of both lenses was higher than one, meaning that the tolerance dose criteria was not accomplished. Also, for the Critical group, a percent score difference of $9 \%$ can be observed between the two plans.

For patient \#2, the best global plan score was obtained with the non-coplanar plan $i 9 n c$ with a percent difference score of $-5 \%$ from the coplanar plan i9c, that presented

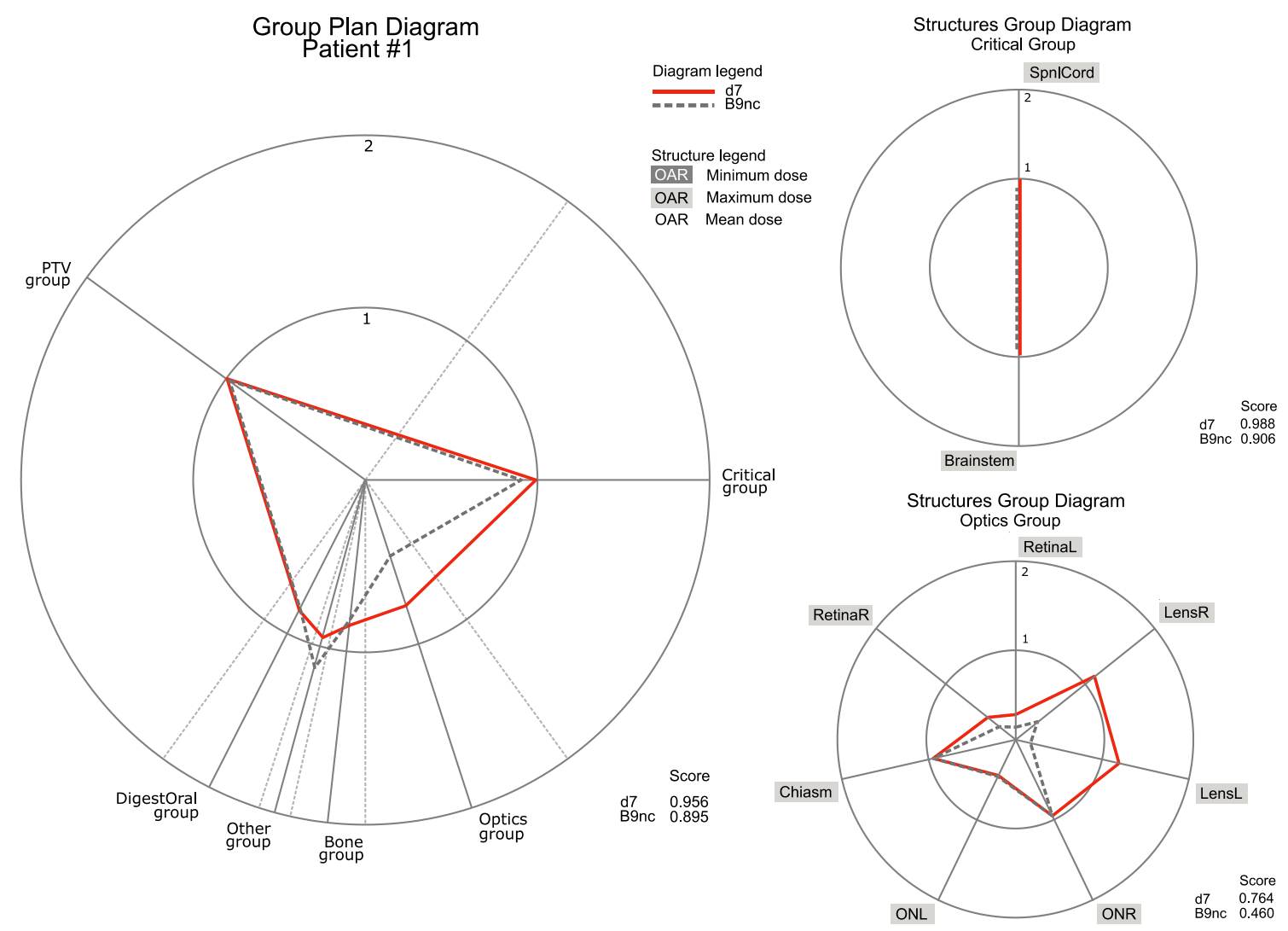

Fig. 1. SPIDERplan group plan diagram of patient \#1 and structures group diagrams for Critical and Optics groups for 7 equidistant beam angles $(d 7)$ and 9 non-coplanar beams plan of algorithm $B(B 9 n c)$. 


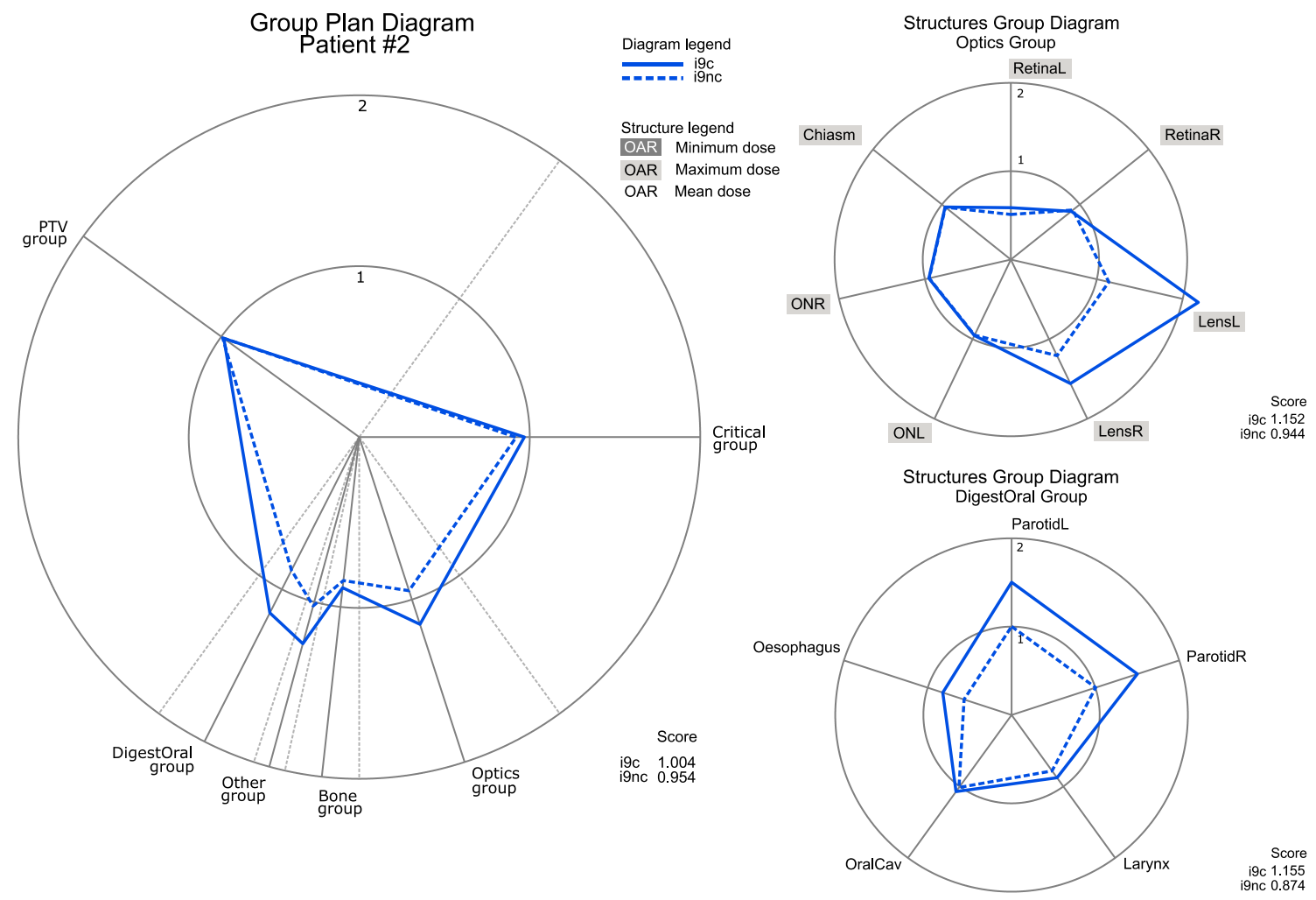

Fig. 2. SPIDERplan group plan diagram of patient \#2 and structures group diagrams for Optics and DigestOral groups for algorithm $i$ with 9 coplanar beams $(i 9 c)$ and 9 non-coplanar beams $(i 9 n c)$

a score of 1.004 (Fig. 2). Also for $i 9 c$, score values higher than one were achieved by the Optics group, the DigestOral group and the Other group, while for $i 9 n c$ only the Other group score was out of tolerance. The reason for these global results was found in the SGDs. Both plans presented scores very near the tolerance or out of tolerance for the lenses (Optics group SGD), for the parotids and for the oral cavity (DigestOral group SGD). Nevertheless, plan $i 9 n c$ was much better than $i 9 c$ plan, since for the mentioned structures it presented score values near one or just slightly higher than one, while $i 9 c$ got score values well above the defined tolerances.

Fig. 3 compares the quality of $B 5 n c$ and $i 9 c$ plans for patient \#3. Globally, plan B5nc attained the best global plan score with a percent global score difference of $-4 \%$ compared to $i 9 c$ plan. This result is obtained even if for the DigestOral group B5nc plan is worse than plan $i 9 c$, due to the relatively lower weight of this group. The main reason for the best score of plan $B 5 n c$ is the almost vanishing scores presented for the optics structures (dotted line at the Optics SGD centre). The topographic relation of the PTV and the optics structure, well separated for this patient, explains this result. 


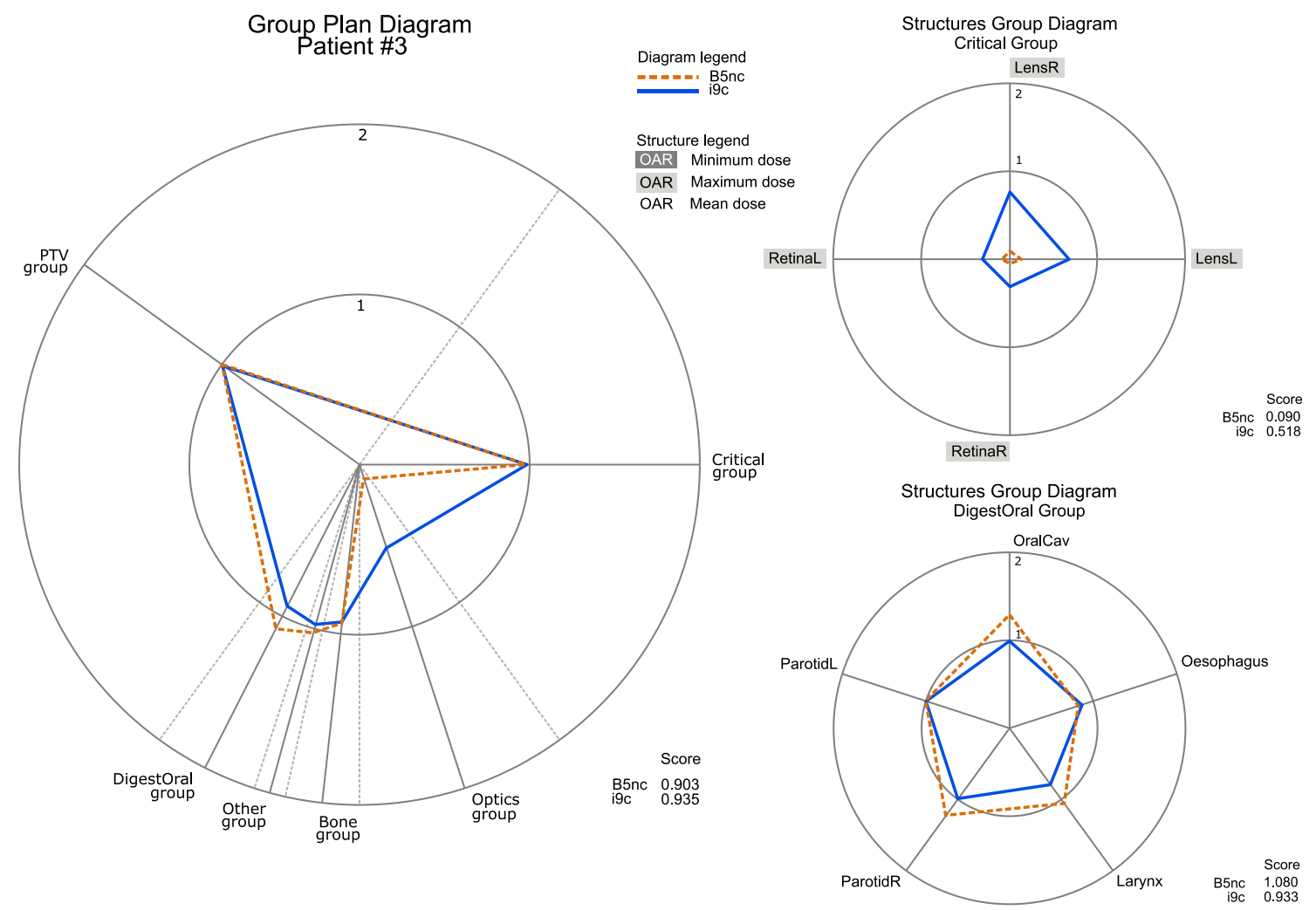

Fig. 3. SPIDERplan group plan diagram of patient \#3 and structures group diagrams for Optics and DigestOral groups for algorithm $B$ with 5 non-coplanar beams (B5nc) and for algorithm $i$ with 9 coplanar beams (i9nc)

\section{Discussion}

In this study, patient specific plan assessment was used to highlight the advantages of BAO for head-and-neck cases.

Among a set of forty NPC clinical cases, three patients were chosen. For patient \#1, important plan quality improvements were achieved when the plan using optimized directions was compared with the equidistant beam angle solution, the standard beam configuration used in the clinical routine. For this patient, the plan generated with BAO presented non-coplanar beam incidences and a higher number of beams than equidistant beam angle solution. This has improved the quality of dose distribution by further sparing important OARs.

The utilization of non-coplanar beams in RT is a very popular approach among medical physicists and mathematicians. There is an intrinsic perception that noncoplanar beam incidences may enhance the quality of the plan. BAO has a crucial role in the selection process of the best beam arrangement, due to the high number of 
possible combinations and geometry complexity. Promising results with non-coplanar BAO have been achieved for different pathology sites such as: lung, liver, head-andneck and intracranial tumours [4,12,13,14]. Nevertheless, depending on site complexity, a patient-by-patient assessment may be required to fully evaluate the real impact of the achieved improvements. For patient $\# 2, i 9 c$ and $i 9 n c$ plans were compared enhancing the advantage of non-coplanarity. The higher score group differences were found for the Optics group (lenses and left retina) and for the DigestOral group (oesophagus, parotids and larynx), where the structures were located very near or even contiguous to the PTVs.

The number of beams needed to achieve a 'good' dose distribution is also an interesting topic due to treatment delivery efficiency reasons. The planner intuition will always predict that plan quality will tend to increase with the number of beams available for optimization. However, the amount of fluence modulation caused by the addition of new beams has a physical threshold, where beyond that level no further plan quality improvements occur. BAO has then an important role to play, achieving better treatment plans with a smaller number of beams. This scenario occurred for patient \#3 where plan $B 5 n c$ was better than $i 9 c$, due to the significant improvement achieved in the optics structures that were completely spared when using fewer non-coplanar beam orientations.

For all patients, PTV group scores were always below one. The high target coverage index may be related with the quality of the FMO and to the definitions of the multicriterial IMRT optimization engine wish-list where a primordial importance was assigned to the PTVs. Also, for the PTV group, small score differences were obtained among the compared plans. For the OARs, the largest score group differences were obtained for the Critical group (patient \#1), for the Optics group (patients \#1, \#2 and \#3) and for the DigestOral group (patients \#2 and \#3), that contained structures of vital importance, as the spinal cord and the brainstem, and with great impact in the patient quality of life, as the lens, the optical nerves, the parotids or the oral cavity. These findings help to support two key aspects: the main contribution of BAO was in further sparing the OARs while maintaining good PTV coverage.

The use of BAO never worsens the treatment plan quality though for some patients, the improvements achieved may be negligible. However, if BAO can be done without the planner's intervention, then it is worth to be considered for all patients, since some of them will greatly benefit from this optimization.

The potential enhancements that an efficient BAO, made in reasonable computing times, may bring to the quality of static IMRT plans, can reopen the discussion about what is the most appropriate inverse planning technique for a given patient treatment.

\section{Conclusions}

In this study the benefits of BAO for head-and-neck patients were addressed. Patient specific analysis of NPC cases were used to highlight the improvements achieved by plans with BAO when compared with IMRT plans using equidistant beam angle directions. Furthermore, the advantages of non-coplanar over coplanar beam plans and 
the importance of BAO in the generation of plans with a small number of beams was highlighted. This study also showed that BAO did not compromise the PTV coverage and that the most important contribution was in the sparing of the normal tissues, taking into account the anatomy specificities of each patient.

\section{Acknowledgments}

The authors would like to express their gratitude to Sebastiaan Breedveld and Ben Heijmen for making available the multicriterial optimization of IMRT plans system Erasmus-iCycle and their valuable support and coaching along the work.

This work was supported by project grant POCI-01-0145-FEDER-028030 and by the Fundação para a Ciência e a Tecnologia (FCT) under project grant UID/Multi/00308/2019.

No potential conflict of interest nor any financial disclosures must be declared.

\section{References}

1. ICRU. International Commission on Radiation Units and Measurements: Prescribing, Recording, and Reporting Photon-Beam Intensity-Modulated Radiation Therapy (IMRT). ICRU Report 83, J ICRU, 10(1), 1-106 (2010). doi: 10.1093/jicru/10.1.Report83

2. Thieke C., Küufer K.H., Monz M. et al.: A new concept for interactive radiotherapy planning with multicriteria optimization: First clinical evaluation. Radiother and Oncol, 85(2), 29298 (2007). doi: 10.1016/j.radonc.2007.06.020

3. Das S.K., Marks L.B.: Selection of coplanar or noncoplanar beams using three-dimensional optimization based on maximum beam separation and minimized nontarget irradiation. Int J Radiat Oncol Biol Phys, 38(3), 643-55 (1997). doi: 10.1016/S0360-3016(97)89489-8

4. Rocha H., Dias J., Ventura T., Ferreira B., Lopes M.C.: A derivative-free multistart framework for an automated noncoplanar beam angle optimization in IMRT. Med Phys, 43(10). 5514-26 (2016). doi: 10.1118/1.4962477.

5. Ehrgott M., Holder A., Reese J.: Beam selection in radiotherapy design. Linear Algebra Appl, 428(5), 1272-312 (2008). doi: 10.1016/j.laa.2007.05.039

6. Craft D.: Local beam angle optimization with linear programming and gradient search. Phys Med Biol, 52(7), 127-35 (2007). doi: 10.1088/0031-9155/52/7/N02

7. Dias J., Rocha H., Ferreira B.C. et al.: Simulated annealing applied to IMRT beam angle optimization: A computational study. Physica Med, 31(7), 747-56 (2015). doi: 10.1016/j.ejmp.2015.03.005

8. Bangert M., Unkelbach J.: Accelerated iterative beam angle selection in IMRT. Med Phys 2016, 43(3), 1073-82 (2016). doi: 10.1118/1.4940350

9. Rocha H., Dias J., Ferreira B.C. et al.: Beam angle optimization for intensity-modulated radiation therapy using a guided pattern search method. Phys Med Biol, 58(9), 2939-53 (2013). doi: 10.1088/0031-9155/58/9/2939

10. Breedveld S., Storchi P., Voet P. et al.: iCycle: integrated, multi-criterial beam angle and profile optimization for generation of coplanar and non-coplanar IMRT plans. Med Phys, 39(2), 951-63 (2012). doi: 10.1118/1.3676689 
11. Ventura T., Lopes M.C., Ferreira B.C. et al.: SPIDERplan: A tool to support decisionmaking in radiation therapy treatment plan assessment. Reports Pract Oncol Radiother, 21(6), 508-16 (2016). doi: 10.1016/j.rpor.2016.07.002

12. Dong P., Lee P., Ruan D et al.: $4 \pi$ Non-Coplanar Liver SBRT: A Novel Delivery Technique. Int J Radiat Oncol Biol Phys, 85(5), 1360-66 (2013). doi: 10.1016/j.ijrobp.2012.09.028

13. Bangert M., Ziegenhein P., Oelfke U.: Comparison of beam angle selection strategies for intracranial IMRT. Med. Phys., 40(1), 011716 (2013). doi: 10.1118/1.4771932

14. Lyu Q., Yu V.I., Ruan D.: A novel optimization framework for VMAT with dynamic gantry couch rotation. Phys Med Biol, 63(12), 125013 (2018). doi: 10.1088/1361-6560/aac704 